\title{
Chronic thyroiditis in lateral ectopic thyroid mimicking cervical metastasis of thyroid cancer
}

\author{
Simone Pederzoli1,2, Tiziana Salviato ${ }^{3}$, Francesco Mattioli4, Gianluca Di Massa ${ }^{3}$ and \\ Giulia Brigante ${ }^{1,2}$
}

1 Unit of Endocrinology, Department of Medical Specialties, Azienda Ospedaliero-Universitaria of Modena, Modena, Italy, 2Unit of Endocrinology, Department of Biomedical, Metabolic and Neural Sciences, University of Modena and Reggio Emilia, Modena, Italy, ${ }^{3}$ Department of Medical and Surgical Sciences for Children and Adults, University of Modena and Reggio Emilia, Modena, Italy, and ${ }^{4}$ Otolaryngology, Head and Neck Surgery Department, Azienda Ospedaliero-Universitaria of Modena, Modena, Italy

Correspondence should be addressed to G Brigante

Email

giulia.brigante@unimore.it

\section{Summary}

We present the case of a 45-year-old Caucasian woman who attended the Endocrinology Unit for a left cervical mass discovered during follow-up for autoimmune chronic thyroiditis. The ultrasound-guided fine-needle aspiration biopsy of the lesion was consistent with a metastasis of follicular thyroid carcinoma. The sonographic neck evaluation revealed no thyroid nodules but three markedly hypoechoic and highly vascularized areas, with irregular margins and hyperechoic spots. In the clinical suspicion of primary thyroid neoplasm, ultrasound-guided fine-needle aspiration biopsy of two of the three areas was performed, but both cytological reports were non-diagnostic, revealing only colloid and blood. Subsequently, the patient underwent surgical removal of the cervical mass, with the intra-operatory consultation with frozen section examination suggesting follicular-like neoplasia. For this reason, thyroidectomy with both central and lateral neck dissection was performed. Surprisingly, the final histologic examination revealed chronic thyroiditis in the thyroid specimen and no evidence of metastasis in the left neck mass. Consequently, the pathological revision of the frozen section assessment led to the final diagnosis of chronic thyroiditis on the lateral ectopic thyroid. This case represents an uncommon example of lateral ectopic thyroid tissue with coexisting normally located thyroid tissue both affected by chronic thyroiditis.

\section{Learning points:}

- Ectopic thyroid must be considered in the diagnostic work-up of lateral neck mass.

- Even if rare, ectopic thyroid tissue can be found lateral to the carotid sheath and with coexisting normally located thyroid tissue.

- As the orthotopic tissue, lateral ectopic thyroid tissue can be affected by chronic thyroiditis, which may complicate the diagnosis both on ultrasound and cytology.

\section{Background}

Ectopic thyroid tissue can be seen in the head and neck and mediastinum including parasitic nodules, and struma ovarii (1). It is characterized by normal thyroid tissue outside the pretracheal area, that is, anterior to the second through the fourth tracheal ring (2). It is a rare condition, with an estimated prevalence ranging from $1 / 100,000$ to 300,000 in the general population, and from $1 / 4,000$ to 8,000 among patients with thyroid diseases $(3,4,5)$. The 
main diagnostic challenge is to distinguish ectopia from metastatic thyroid carcinoma (1).

Ectopic thyroid is likely due to an abnormal organ development of unknown cause during embryogenesis. Thyroid gland originates from two different embryological structures: a major anlage, which is located in the midline (i.e., within the posterior part of the embryonic mouth cavity) and two ultimobranchial bodies, which derive from the fourth pharyngeal pouch and are located symmetrically on the sides of the developing neck (6). In particular, the median anlage gives origin to the thyroid follicular cells, while both the lateral ones give origin to the parafollicular cells (6). At the fourth week of gestation, the median anlage begins to descend from the foramen cecum of the tongue through the thyroglossal duct until it reaches the final position, where it merges with the medially migrating lateral anlages, giving birth to the final gland (6).

Alterations in this process can lead to two types of thyroid ectopia: along the midline or, more rarely, in the lateral neck. In most cases, a failure in descent or migration of the median anlage occurs, and the result is the presence of a lingual thyroid, a midline ectopic thyroid, or a thyroglossal duct cyst $(2,7)$. Less frequently, there is an aberrant lateral migration of the median anlage or a lack of fusion of the lateral anlages with the median one, leading to lateral ectopic thyroid (i.e. in the submandibular area or near the carotid sheath) $(2,7)$.

The molecular mechanism portending to these alterations is not completely clear, although mutations in transcription factor genes involved in the process of thyroid organogenesis and migration, such as thyroid transcription factor 1 (TTF-1), NK2 homeobox (Nkx2), paired box 8 (Pax-8), forkhead box E1 (FOXE1), and hematopoietically expressed homeobox (Hhex) have been claimed $(2,8)$.

Because of their similar clinical presentation, lateral ectopic thyroid raises differential diagnosis challenges, especially with metastatic thyroid cancer (9).

We report a case of ectopic thyroid presenting as a left cervical mass with clinical and pre-operative features suggesting metastatic cancer from the thyroid.

\section{Case presentation}

A 45-year-old Caucasian woman was referred to the Endocrinology Unit by the otolaryngologist for a suspicious thyroid-derived cervical node metastasis in the II left level of Robbins.

Her clinical history revealed an autoimmune chronic thyroiditis without nodules since 1991, treated with replacement doses of levothyroxine (75 $\mu \mathrm{g}$ daily). During follow-up, a marked hypoechoic lesion of $16 \mathrm{~mm}$ in the II left level of Robbins was firstly detected at neck ultrasound in 2017. Either lipoma or an enlarged reactive lymph node was suspected, hence instrumental follow-up was advised.

About 2 years after the initial assessment, an ultrasound-guided fine-needle aspiration (FNA) biopsy was performed due to the growth of the lesion. Microscopic examination showed small lymphocytes and epithelial nests with rare follicular-like features composed of cells with eosinophilic cytoplasm and irregular nuclei. The overall features were deemed consistent with metastasis of follicular thyroid carcinoma. Thus, an endocrinological evaluation was recommended.

\section{Investigation}

The patient had a family history of thyroid disease; her mother was affected by autoimmune chronic thyroiditis and multinodular goiter. The remainder history was unremarkable except for treatment with levothyroxine.

The patient referred no fever, pain, dysphagia, voice changes, or respiratory complaints, and the neck physical examination was unremarkable; in particular, there was no evidence of palpable nodules both in the thyroid and laterocervical regions. Slightly increased values of thyroidstimulating hormone (TSH) $(6.24 \mu \mathrm{IU} / \mathrm{mL}$, reference range $0.27-4.20 \mu \mathrm{IU} / \mathrm{mL}$ ), with normal levels of free thyroxine (fT4) (11 pg/mL, reference range 7-15 pg/mL) suggested an underdosing of the ongoing replacement therapy, which required adjustment.

The sonographic neck evaluation revealed a thyroid of normal size and location, with inhomogeneous structure and fibrous branches typical of chronic thyroiditis (Fig. 1). No nodular lesions were clearly detectable, but three areas were reported (one in the upper third of the right lobe of $27 \mathrm{~mm}$ and two in the middle third of the left lobe, respectively the anterior one of $14 \mathrm{~mm}$ and the posterior one of $12 \mathrm{~mm}$ ). All of these areas were markedly hypoechoic, highly vascularized, with irregular margins and hyperechoic spots. Moreover, a markedly hypoechoic and highly vascularized lesion of $19 \mathrm{~mm}$ without evident germinal center was confirmed at the II left level of Robbins (Fig. 2).

Due to the clinical suspicion of primary thyroid neoplasm, ultrasound-guided FNA biopsy of two of the three intra-thyroid hypoechoic areas was performed; both cytological reports were non-diagnostic, revealing only colloid and blood (category Thy1 according to Bethesda System) (10). FNA was repeated on the left cervical lesion; 


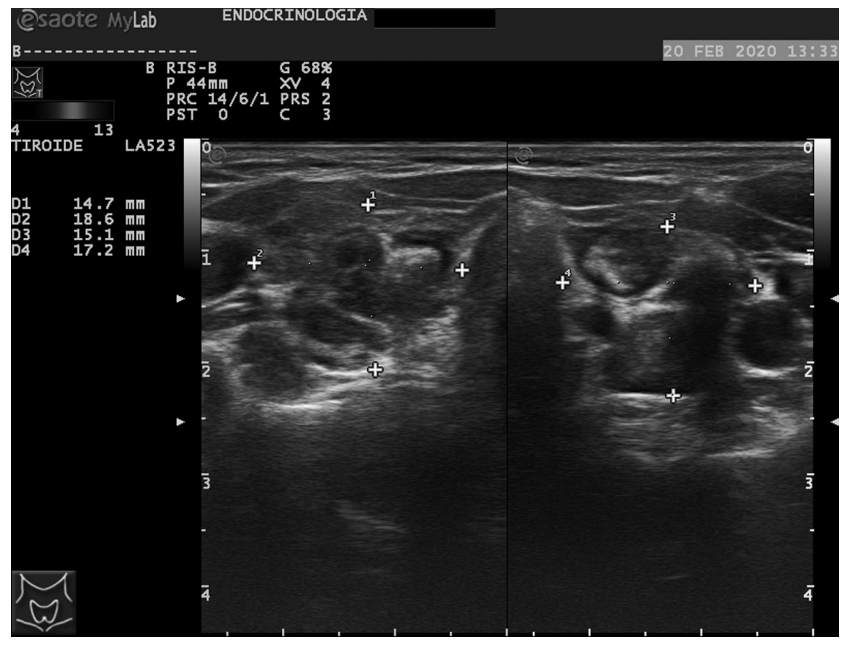

\section{Figure 1}

Ultrasound image of the normally located thyroid gland, presenting normal dimensions and diffuse inhomogeneity, compatible with thyroiditic pattern.

the cytology highlighted three small epithelial-like cell nests with enlarged nuclei, and thyroglobulin concentration in washout fluid (FNA-Tg) was very high ( $>487 \mathrm{ng} / \mathrm{mL}$ ), suggesting the presence of thyroid cells, as it happens in thyroid-derived node metastasis and less frequently in benign ectopic thyroid (i.e. parasite or intranodal inclusion) (1). Thus, a further FNA biopsy was performed on the same thyroid areas, yielding non-diagnostic material.

Finally, a cervical MRI was performed, confirming the presence in the left laterocervical region of an oval, solid, and well-defined lesion of $19 \mathrm{~mm}$, in close relation to the sternothyroid muscle (Fig. 3A and B). This lesion

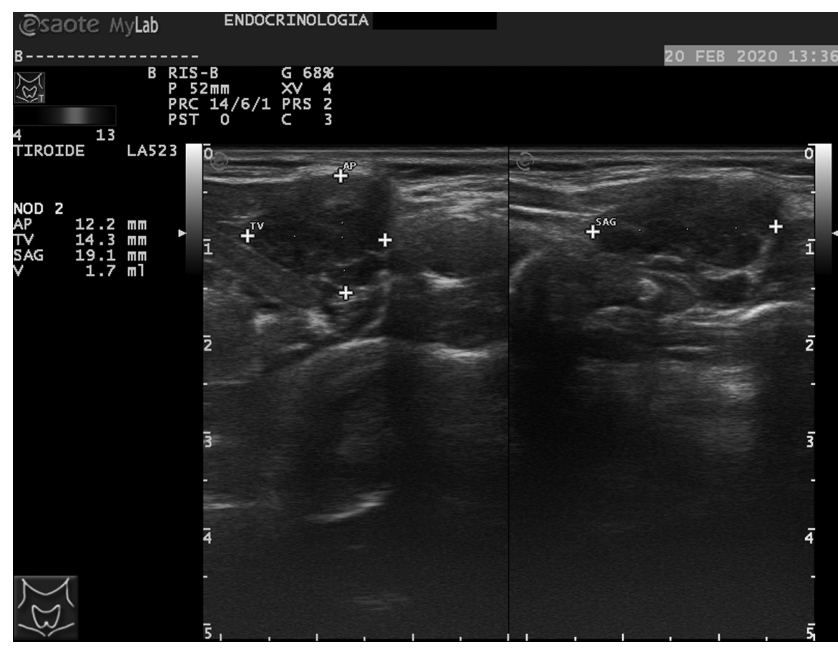

Figure 2

Ultrasound image of the lesion at the II left level of Robbins (on the left, the anteroposterior and transverse diameters, on the right the longitudinal diameter). had a medium to high signal in T1-T2 and homogeneous enhancement after contrast agent injection and was described as compatible with pathologic adenopathy.

\section{Treatment}

The patient underwent resection of the left laterocervical mass. An intra-operatory consultation with frozen section examination suggested follicular-like neoplasia (Fig. 4A and $\mathrm{B})$. Thus, total thyroidectomy with both central and

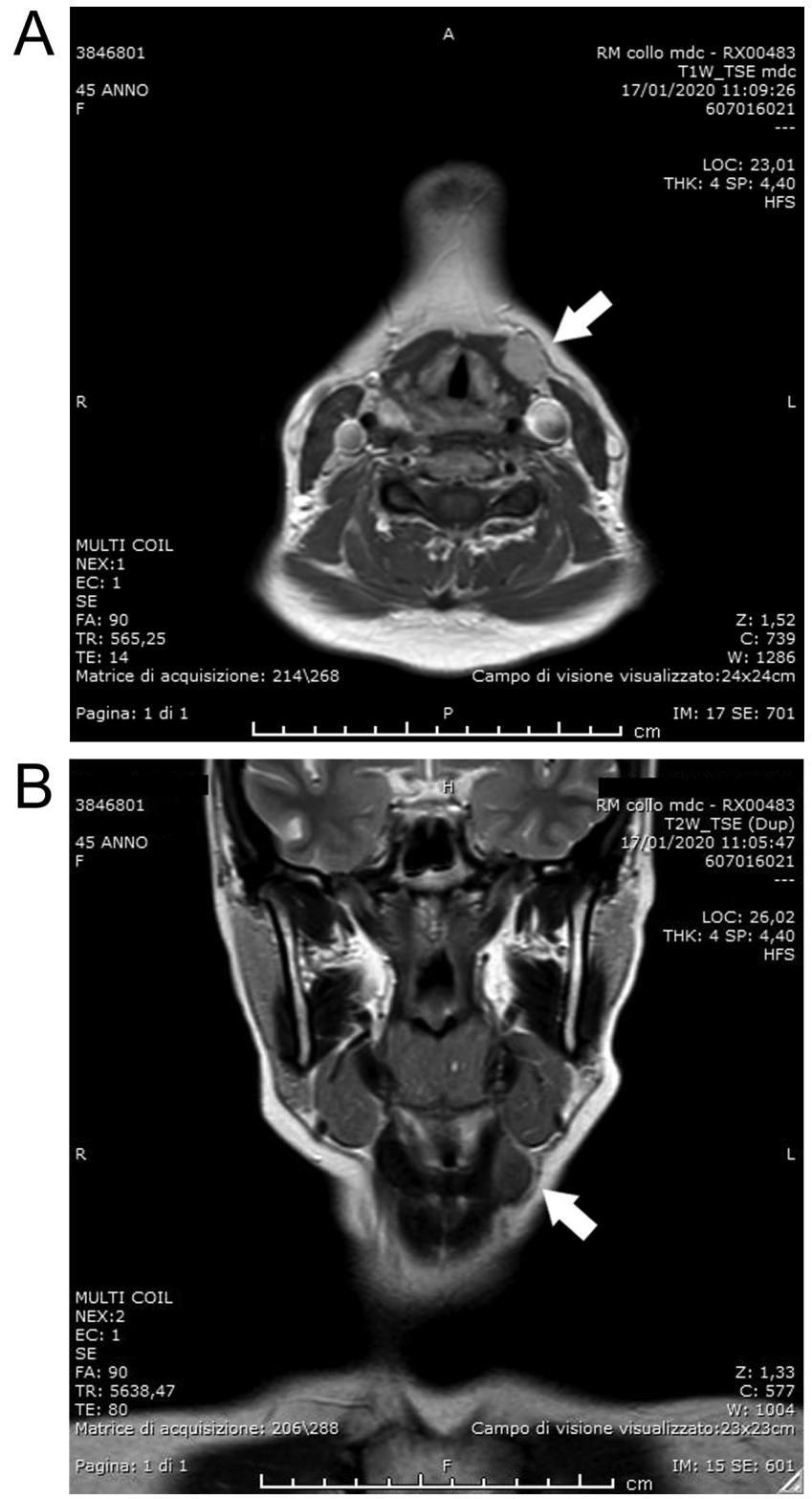

Figure 3

Axial $\mathrm{T}_{1}$-weighted MRI image post contrast of the lesion at the II left level of Robbins (A). Coronal T2-weighted MRI image of the lesion at the II left level of Robbins (B). 


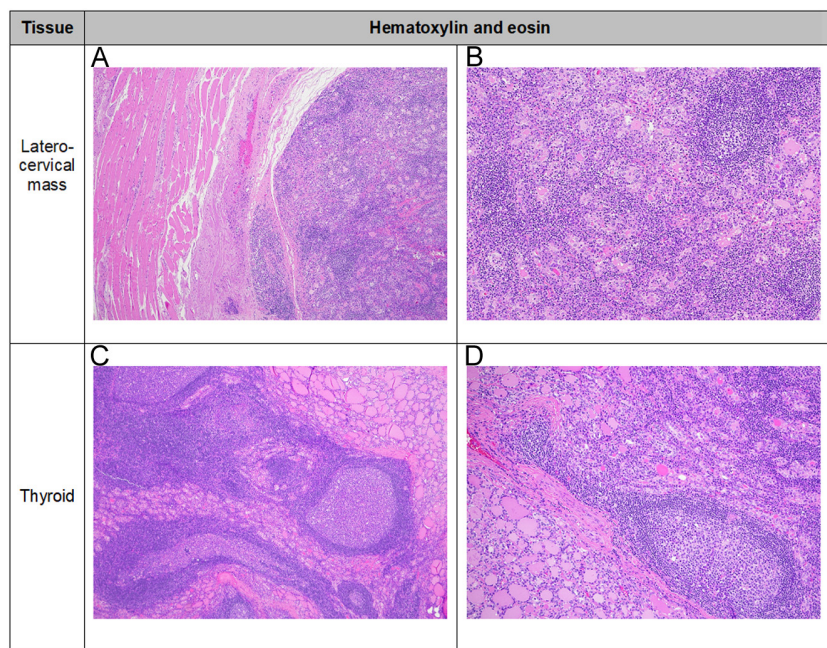

Figure 4

(A) Laterocervical mass hematoxylin eosin (HE) 4x: laterocervical mass with subverted structure due to the presence of glandular elements; (B) laterocervical mass HE 10x: detail of the previous image at higher magnification; (C) thyroid HE 4x: lymphocytic thyroiditis where residual thyroid parenchyma and marked inflammatory infiltrate can be seen; (D) thyroid HE 10x: detail of the previous image at higher magnification.

lateral left neck dissection was carried out. Microscopic examination of formalin-fixed and routinely processed tissue showed chronic thyroiditis with hyperplastic lymphoid follicles, Hurthle-cell metaplasia, and reactive fibrosis (Fig. 4C and D). There was no microscopic evidence of metastasis within the left neck dissection specimen. For this reason, a pathological revision of the frozen section assessment was carried out, revealing marked inflammatory tissue disrupting the follicular architecture and leading to the final diagnosis of chronic thyroiditis on the lateral ectopic thyroid. At the immunohistochemical staining in the laterocervical mass, a positivity for TTF-1 (Fig. 5A) and thyroglobulin (Tg) (Fig. 5B), was found.

\section{Outcome and follow up}

After surgery, the patient continued levothyroxine replacement therapy increasing the dose up to $125 \mu \mathrm{g}$ daily and started calcium-vitamin D integration, which was stopped after few days for a rapid recovery of serum calcium levels. However, laryngoscopy after surgery showed reduced motility of the left vocal cord, and the patient developed dysphonia, which required logopedic rehabilitation.

At the last follow-up evaluation, 3 months after surgery, the biochemical assessment documented undetectable serum $\mathrm{Tg}(<0.1 \mathrm{ng} / \mathrm{mL})$, with slightly detectable anti-Tg antibodies $(7 \mathrm{IU} / \mathrm{mL}$, reference range $<4 \mathrm{IU} / \mathrm{mL})$. Neck ultrasound revealed only avascular small hypoechoic area of $7 \mathrm{~mm}$ in left thyroid lodge, compatible with normal thyroid

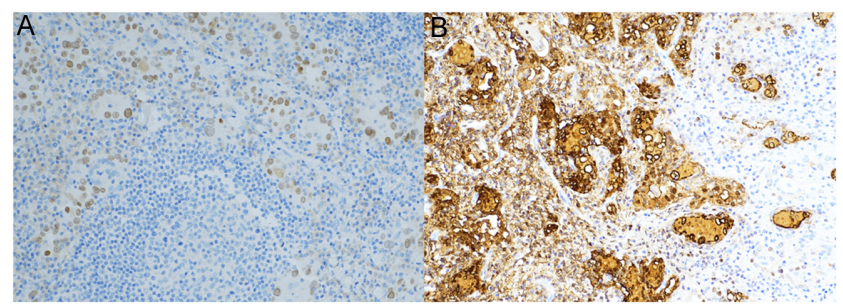

\section{Figure 5}

Immunohistochemical staining in the laterocervical mass: (A) thyrocytes positive for thyroid transcription factor-1 (TTF-1); (B) thyroid follicles positive for anti-thyroglobulin antibody, specific marker of thyroid differentiation.

tissue, even if the scan was limited by the presence of evolving scar tissue due to short time from surgery. Moreover, for the detection of subclinical hypothyroidism (TSH $7.51 \mu \mathrm{IU} / \mathrm{mL}$, fT4 $9.1 \mathrm{pg} / \mathrm{mL}$ ), levothyroxine increase was needed, up to $135 \mu \mathrm{g}$ daily. Finally, a normal phospho-calcic profile was detected, confirming the complete recovery of parathyroid functionality, and dysphonia had been significantly improving, thanks to logopedic rehabilitation.

\section{Discussion}

The present case represents an uncommon example of thyroiditis occurring in both normally located and lateral ectopic thyroid tissue.

Lateral location occurs in only 1-3\% of thyroid ectopia, usually in the submandibular area (70\%) or at least medial to the carotid sheath $(30 \%)(2,9)$. In our case, the ectopic tissue was lateral to the carotid sheath, at the second left level of Robbins. Moreover, our patient also had thyroid gland of normal dimension in the pre-tracheal area, while $60 \%$ of lateral thyroid ectopia represents the only thyroid tissue in the body $(2,7)$. Finally, both the ectopic and the normally located thyroid tissue were affected by chronic thyroiditis, complicating the diagnosis both on ultrasound and cytology. There are only a few case reports of thyroiditis in ectopic tissue (5), and to our knowledge, only another case describes the concomitant presence of thyroiditis both in normally located and in ectopic, midline, thyroid tissue in a 13-year-old Indian girl $(5,11)$.

Furthermore, the patient did not complain of a neck enlargement, and the mass was discovered incidentally during ultrasound follow-up of the known chronic thyroiditis. Usually, ectopic masses - in the absence of normally localized thyroid - are diagnosed when they grow consequently to the proliferative stimulus of high TSH levels $(2,8,12)$.

The cytological result was suspicious of a thyroidderived metastasis due to both lymphocytes and follicular 
cells with nuclear irregularities (13). With the benefit of hindsight, such alterations were secondary to the ongoing chronic thyroiditis; in fact, in that particular condition, follicular cells may mimic some structural and nuclear features of papillary carcinoma (i.e. papillary pattern and nuclear grooves), hence raising diagnostic issues in the differential diagnosis (14). On the other hand, the lymphocytic background seen in cytological smears merely reflects the inflammatory tissue typical of chronic thyroiditis.

These findings, along with the frozen section assessment suggesting follicular-like neoplasia warranted a more invasive surgery, but they were not confirmed on paraffin, permanent sections. As a complication of surgical treatment, a transient-reduced motility of the left vocal cord occurred. Thus, the patient has been overtreated, due to misdiagnosis of an extremely rare pathological condition. However, we also have to consider that the patient was already on replacement therapy for hypothyroidism and that malignancy has been reported in about $12 \%$ of lateral ectopic thyroid (2).

In conclusion, ectopic thyroid enters the diagnostic work-up of lateral neck mass, especially in subjects without nodular alterations of the orthotopic thyroid. Even if rare, lateral ectopic tissue can be affected by chronic thyroiditis resulting in challenging ultrasound scan and cytopathologic evaluation. A comprehensive clinical history of the patient is of critical importance in patient assessment, therapy and follow-up.

\section{Declaration of interest}

The authors declare that there is no conflict of interest that could be perceived as prejudicing the impartiality of the research reported.

\section{Funding}

This research did not receive any specific grant from any funding agency in the public, commercial or not-for-profit sector.

\section{Patient consent}

Written informed consent for publication of her clinical details was obtained from the patient.

\section{Author contribution statement}

S P and G B wrote the manuscript and were involved in the endocrine care of the patient. F M performed the neck surgery on the patient. T S and D $\mathrm{G}$ provided histological images and performed the pathological analyses.

\section{References}

1 Barbieri A, Prasad ML \& Gilani SM. Thyroid tissue outside the thyroid gland: differential diagnosis and associated diagnostic challenges. Annals of Diagnostic Pathology 202048 151584. (https://doi. org/10.1016/j.anndiagpath.2020.151584)

2 Prado H, Prado A \& Castillo B. Lateral ectopic thyroid: a case diagnosed preoperatively. Ear, Nose, and Throat Journal 201291 E14-E18. (https:// doi.org/10.1177/014556131209100417)

3 Mussak EN \& Kacker A. Surgical and medical management of midline ectopic thyroid. Otolaryngology: Head and Neck Surgery 2007136 870-872. (https://doi.org/10.1016/j.otohns.2007.01.008)

4 Alanazi SM \& Limaiem F. Ectopic thyroid. In StatPearls [Internet]. Treasure Island (FL): StatPearls Publishing LLC, 2020.

5 Garcia-Rodriguez L, Dharia R \& Massey B. Ectopic thyroid tissue with Hashimoto's thyroiditis. WMJ 2016115 47-48.

6 De Felice M \& Di Lauro R. Thyroid development and its disorders: genetics and molecular mechanisms. Endocrine Reviews 200425 722-746. (https://doi.org/10.1210/er.2003-0028)

7 Feller KU, Mavros A \& Gaertner HJ. Ectopic submandibular thyroid tissue with a coexisting active and normally located thyroid gland: case report and review of literature. Oral Surgery, Oral Medicine, Oral Pathology, Oral Radiology, and Endodontics 200090 618-623. (https:// doi.org/10.1067/moe.2000.108804)

8 Nasiru Akanmu I \& Mobolaji Adewale O. Lateral cervical ectopic thyroid masses with eutopic multinodular goiter: an unusual presentation. Hormones 20098 150-153. (https://doi.org/10.14310/horm.2002.1232)

9 Choi JY \& Kim JH. A case of an ectopic thyroid gland at the lateral neck masquerading as a metastatic papillary thyroid carcinoma. Journal of Korean Medical Science 200823 548-550. (https://doi.org/10.3346/ jkms.2008.23.3.548)

10 Cibas ES \& Ali SZ. The 2017 Bethesda system for reporting thyroid cytopathology. Thyroid 201727 1341-1346. (https://doi.org/10.1089/ thy.2017.0500)

11 Chopra A, Singh Y, Kaushal M, Taneja A \& Kulshreshtha B. Simultaneous occurrence of thyroiditis in ectopic and eutopic thyroid masquerading as thyroglossal cyst. Journal of Clinical and Diagnostic Research 201711 Od17-Od19. (https://doi.org/10.7860/ JCDR/2017/25192.9897)

12 Hazarika P, Siddiqui SA, Pujary K, Shah P, Nayak DR \& Balakrishnan R. Dual ectopic thyroid: a report of two cases. Journal of Laryngology and Otology 1998112 393-395. (https://doi.org/10.1017/s0022215100140563)

13 Tseng FY, Hsiao YL \& Chang TC. Cytologic features of metastatic papillary thyroid carcinoma in cervical lymph nodes. Acta Cytologica 200246 1043-1048. (https://doi.org/10.1159/000327105)

14 Haberal AN, Toru S, Ozen O, Arat Z \& Bilezikçi B. Diagnostic pitfalls in the evaluation of fine needle aspiration cytology of the thyroid: correlation with histopathology in 260 cases. Cytopathology 200920 103-108. (https://doi.org/10.1111/j.1365-2303.2008.00594.x)

Received in final form 29 May 2021

Accepted 7 June 2021 